\title{
Análise qualitativa e quantitativa da histerese entre vazão e concentração de sedimentos durante eventos hidrológicos
}

\author{
Jean P. G. Minella ${ }^{1}$, Gustavo H. Merten² \& Priscila F. Magnago ${ }^{2}$
}

\begin{abstract}
RESUMO
A relação entre a vazão - Q e a concentração de sedimentos em suspensão - CSS, apresenta padrões temporais complexos durante eventos de chuva governados pela geomorfologia, uso e manejo do solo e precipitação. Este fato tem implicações relevantes no entendimento e na descrição dos processos erosivos e de produção de sedimentos em bacias, padrões que podem ser avaliados pelas características da histerese entre a CSS e a Q. Neste estudo foi analisado o efeito histerese de 19 eventos de chuva-vazão em uma bacia agrícola, no sul do Brasil; os eventos foram separados em duas fases distintas em relação ao manejo do solo, com e sem a utilização de práticas de conservação de solos. O conjunto de eventos foi classificado segundo o tipo de histerese e calculado o valor de um índice de histerese (IH) para avaliar a magnitude desta defasagem. Os resultados mostram que o padrão de histerese foi de laço no sentido horário para os dois momentos, representando uma rápida mobilização e transferência de sedimentos na bacia. Em relação ao IH verificou-se que, apesar de não diferir estatisticamente entre os dois momentos, existem evidências de que as práticas de conservação de solos influenciaram sua variabilidade.
\end{abstract}

Palavras-chave: bacia hidrográfica, monitoramento ambiental, hidrossedimentologia

\section{Qualitative and quantitative analysis of hysteresis between sediment concentration and flow rate during hydrologic events}

\begin{abstract}
The relationship between flow rate - $\mathrm{Q}$ and suspended sediment concentration - SSC demonstrates complex temporal patterns during hydrologic events, which are governed by geomorphology, land use, soil management and rainfall. This fact has important implications for understanding and describing erosive processes and sediment production in catchments. These patterns can be evaluated by their loop hysteresis characteristics on the graph of SSC vs. Q. In this study, the hysteresis effect was analyzed for 19 rainfall events in a rural catchment in Southern Brazil. The events were separated in two groups in relation to soil management (with and without soil management practices). The events were classified according to the type of hysteresis, and a hysteresis index $(\mathrm{HI})$ was calculated to evaluate the magnitude of the difference in phase. Results show that the hysteresis pattern was a clockwise loop, indicating that the peak SSC occurred before the peak $Q$, which represents rapid mobilization and transfer of sediment in the catchment. The HI was not statistically different between the two periods studied, although there is evidence that the conservation practices do have some influence on the index.
\end{abstract}

Key words: catchment, environmental monitoring, hydrosedimentology 


\section{INTRODUÇÃO}

A erosão é definida como o processo de desagregação e remoção de partículas do solo pela ação combinada do impacto da gota de chuva e da ação do escoamento superficial (Morgan, 2005). Além dos impactos na degradação do solo, o processo erosivo controla os impactos dos sedimentos erodidos nos recursos hídricos. Os problemas podem ser de natureza física, pela presença de grandes volumes de sedimentos que provocam o assoreamento dos rios e água, causando enchentes e dificultando a navegação. Esses impasses também podem ser de natureza química e biológica, pelo carreamento de poluentes e patógenos para os rios adsorvidos aos sedimentos. Portanto, o conhecimento da dinâmica do processo erosivo na escala de bacias é de fundamental relevância para o gerenciamento dos recursos hídricos. Neste contexto, a produção de sedimentos tem sido utilizada como indicador dos impactos das atividades antrópicas aos recursos hídricos (Lacombe et al., 2008; Walling, 2008; Nyssen et al., 2009; Horowitz, 2009).

Através do monitoramento da Q e da CSS e da elaboração de hidrogramas (gráficos de variação da vazão - $Q$ com o tempo) e de sedimentogramas (gráficos de variação da CSS com o tempo) é possível estudar a variabilidade temporal dessas duas variáveis. A partir da forma do hidrograma, da forma do sedimentograma e da relação existente entre a Q e a CSS, é possível inferir sobre o comportamento hidrossedimentológico de uma bacia (Klein, 1984; Williams, 1989; Jansson, 2002; Stott, 2006; Lefrancois et al., 2007). Os processos que determinam a distribuição da CSS variam de acordo com as condições relacionadas com os processos erosivos predominantes e da capacidade de transporte dos sedimentos (Walling, 1999; Minella et al., 2009). Assim, os valores da CSS na fase de ascensão do hidrograma são diferentes dos valores para a mesma vazão, durante a recessão.

Este artigo teve como objetivo principal classificar e quantificar a histerese que ocorre entre a vazão e a concentração de sedimentos em suspensão, durante eventos de precipitação em uma bacia rural caracterizada por processos ativos de erosão em entressulcos e em sulcos e elevada produção de sedimentos. Complementarmente, o estudo discute a variabilidade temporal dos índices de histerese e os fatores que os controlam.

\section{Material E MÉTOdos}

O estudo foi desenvolvido com base nos dados do monitoramento hidrossedimentológico de uma bacia rural de cabeceira com intensa atividade agrícola, grande fragilidade ambiental e significativo impacto aos recursos naturais (solo e água) devido à erosão. Nesta bacia o processo de geração e transferência de sedimentos das vertentes para o canal fluvial ocorre, fundamentalmente, durante os eventos de precipitação, sendo o comportamento da CSS e da Q no tempo do evento o foco de análise. Neste sentido foi utilizada a histerese entre as duas variáveis para caracterizar o comportamento hidrossedimentológico desse tipo de bacia em cuja análise se buscou não apenas caracterizar a histerese qualitativamente, mas também aplicar um índice quantitativo que permitisse a comparação entre eventos e, futuramente, entre bacias. A metodologia foi aplicada em eventos característicos significativos de diferentes épocas e magnitudes, na tentativa de representar o processo e identificar fatores controladores de possíveis diferenças.

\section{Análise qualitativa da histerese}

A interpretação da histerese se inicia com a determinação das séries temporais que discretizam a variação dos valores de Q e da CSS, em função do tempo. Graficando os dados de CSS em função dos dados de $\mathrm{Q}$, tem-se a relação entre as variáveis, caracterizando a curva de histerese (Figura 1).
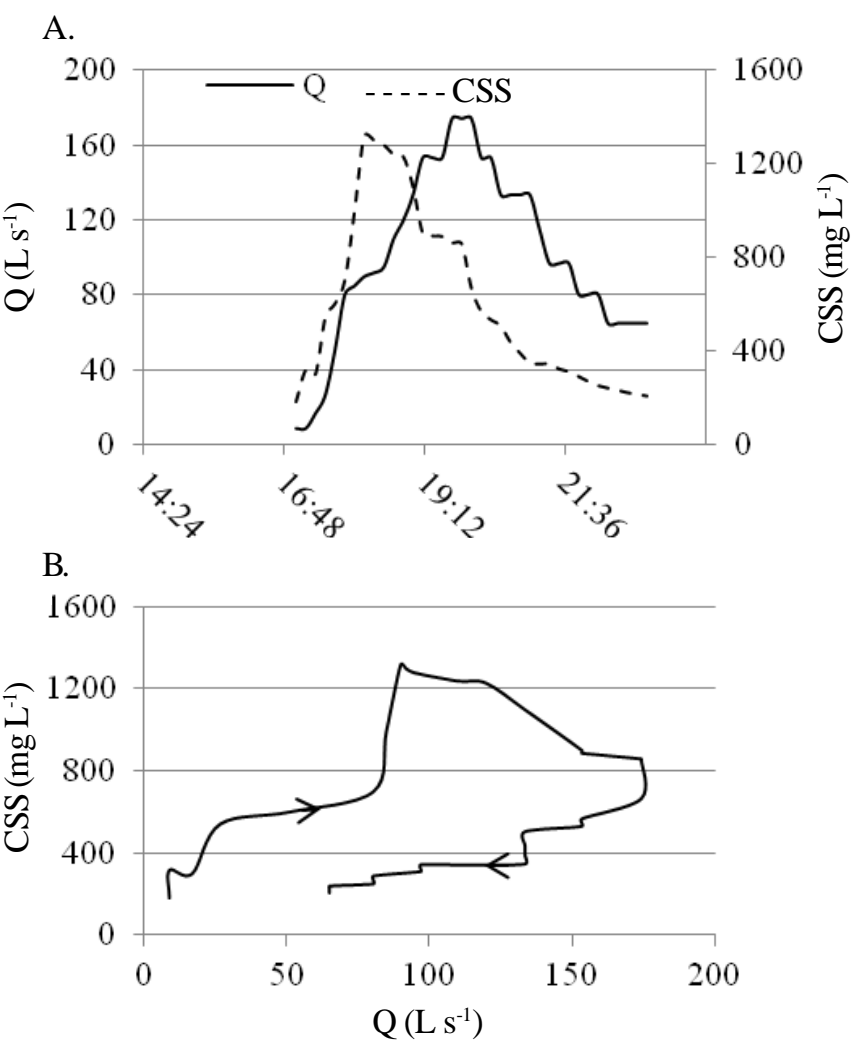

Figura 1. Exemplo de hidrograma, sedimentograma e relação concentração de sedimentos em suspensão (CSS) versus vazão $(Q)$

A histerese é o aparecimento de um atraso na evolução de um fenômeno físico em relação a outro. O gráfico da variação da CSS versus vazão - $Q$, representa a ocorrência do efeito histerese. De maneira geral, a dinâmica da Q e da CSS durante os eventos não apresenta um sincronismo de fase (Lefrancois et al., 2007).

O comportamento hidrossedimentológico da bacia pode ser analisado a partir do sentido e do formato do laço de histerese que sofre influência direta das características apresentadas pelo hidrograma e pelo sedimentograma (Williams, 1989). O laço de histerese pode ter sentido horário, anti-horário ou formato oito, características essas que refletem as diferenças entre os tempos de pico e da declividade dos ramos ascendente e descendente do hidrograma e do sedimentograma. A Figura 2 ilustra essas três classes de curva. 
A.

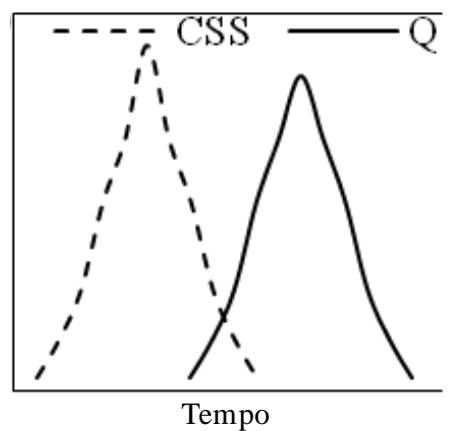

B.

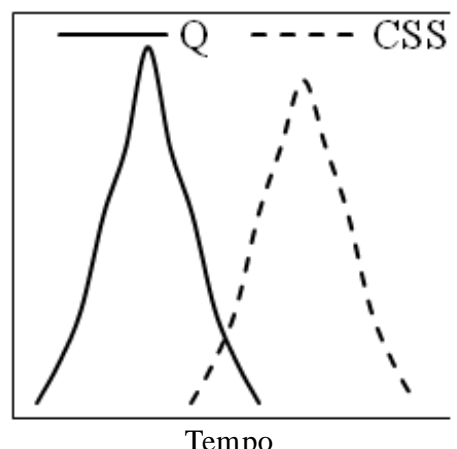

C.

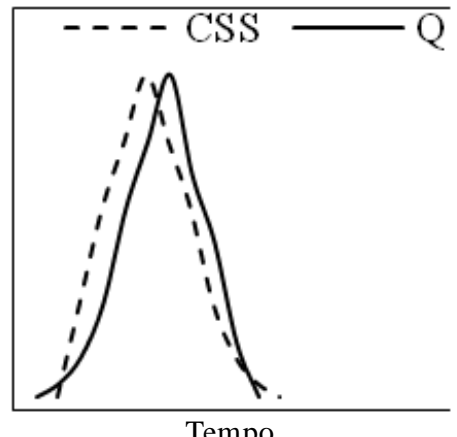

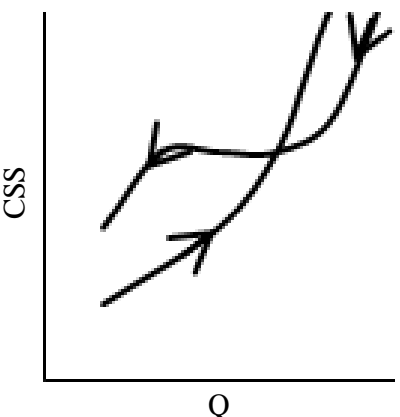

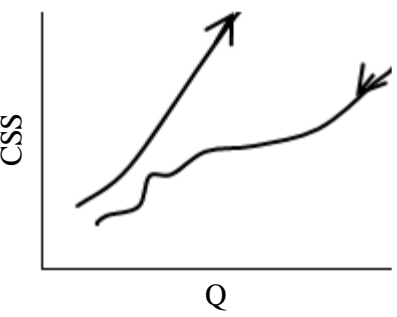

రి

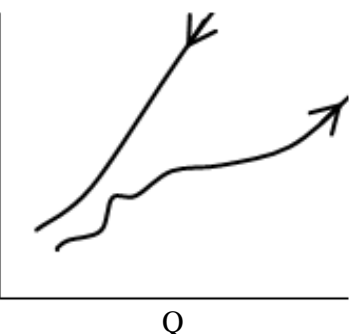

$\mathrm{Q}$

de fontes mais distantes, como aqueles sedimentos que são mobilizados na bacia vertente e transferidos pelo escoamento superficial para o canal fluvial (Lenzi \& Lorenzo, 2000; Goodwing et al., 2003; Orwing \& Smart, 2004).

Quando a curva apresenta tanto o comportamento horário quanto o anti-horário, tem-se um laço com formato em oito. Conforme Williams (1989), isto ocorre quando a CSS e a Q começam a aumentar aproximadamente juntas mas é a CSS que atinge o pico primeiro, produzindo uma curva horária; após a ocorrência do pico da CSS a disponibilidade e o transporte de sedimentos são suficientemente elevados fazendo com que a CSS RD decresça lentamente com o tempo enquanto a Q decresce mais rapidamente ou, em outras palavras, o ramo descendente da $\mathrm{Q}$ é mais declivoso que o ramo descendente da CSS.

\section{Análise quantitativa da histerese}

Além da análise qualitativa é conveniente poder quantificar o comportamento da histerese permitindo a comparação entre eventos ou entre bacias. $\mathrm{O}$ formato do laço de histerese pode ser quantificado a partir da metodologia apresentada por Lawler et al. (2006). O índice de histerese - IH, é obtido a partir da análise dos dados de vazão - Q e CSS e da construção do gráfico de $\mathrm{Q}$ versus CSS; primeiro, conhecendo-se a vazão máxima - $Q_{\max }$ e a vazão inicial mínima - $Q_{\min }$ do evento, calculase o valor central entre a vazão máxima e mínima do ramo ascendente $-Q_{\text {cen }}$ do evento (Eq. 1).

$$
\mathrm{Q}_{\text {cen }}=0,5 *\left(\mathrm{Q}_{\max }-\mathrm{Q}_{\min }\right)+\mathrm{Q}_{\min }
$$

Para o valor central - $Q_{\text {cen }}$ obtido encontram-se os valores de CSS RC e no ramo decrescente a CSS RD utilizando-se o gráfico da CSS vs. Q (Figura 3).

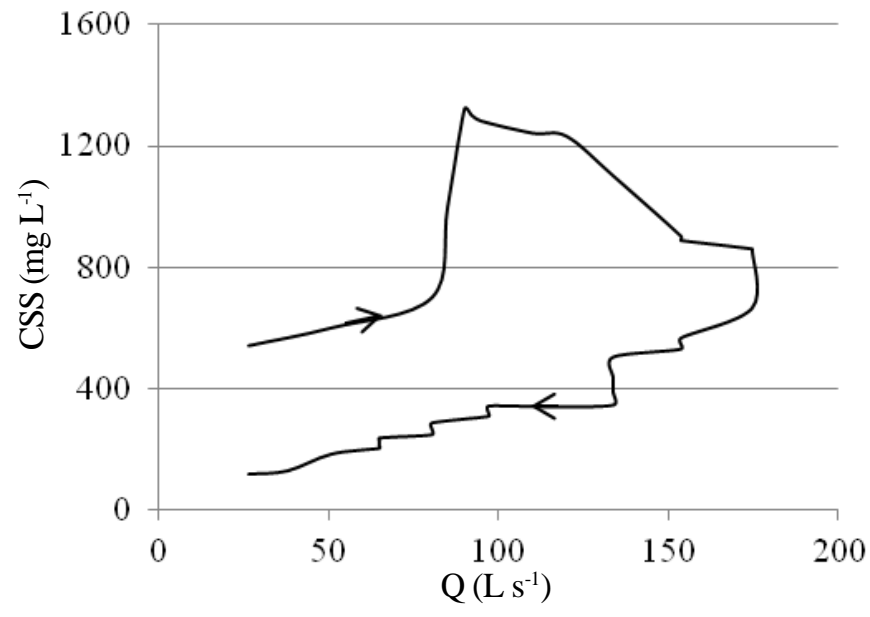

Figura 3. Exemplo de laço de histerese

Os valores de CSS RC e de CSS RD podem ser obtidos do gráfico a partir da interpolação entre os pontos que possuem CSS e Q conhecidas (medidas). Se a curva de histerese possuir sentido horário, o índice de histerese - IH será positivo e calculado pela Eq. 2.

$$
\mathrm{IH}=(\mathrm{CSS} \mathrm{RC} / \mathrm{CSS} \mathrm{RD})-1
$$


e, se a curva de histerese possuir sentido anti-horário, o índice de histerese - IH, será negativo e calculado pela Eq. 3 .

$$
\mathrm{IH}=(-1 /(\mathrm{CSS} \mathrm{RC} / \mathrm{CSS} \mathrm{RD}))+1
$$

O valor do índice de histerese - $\mathrm{IH}$, tende a reduzir durante uma sequência de eventos devido ao aumento do conteúdo de água no solo, determinando que novas fontes de sedimentos sejam incorporadas a toda a bacia vertente (Lawler et al., 2006). Outra razão é a intensificação da atividade agrícola que condiciona a um aumento da disponibilidade de sedimentos pelo revolvimento do solo e geração de depósitos próximos e dentro do canal fluvial (Bowes et al., 2005). Para ambos os casos a magnitude da CSS aumenta em comparação com uma situação de eventos esparsos e com bacias com cobertura do solo permanente e em condições em que ocorrem eventos chuvosos em sequência e/ou em bacias com significativa alteração do uso para a agricultura, aumentando a disponibilidade de sedimentos para ser erodido e transportado e, consequentemente, a magnitude da CSS para uma mesma faixa de vazões.

\section{Área de estudo}

A análise qualitativa e quantitativa da histerese foi efetuada para eventos ocorridos em uma bacia do arroio Lajeado Ferreira, município de Arvorezinha, RS (28 52'S e 52 05'O). A bacia possui $1,19 \mathrm{~km}^{2}$ de área de drenagem e se localiza na região centronorte do Rio Grande do Sul. O clima na bacia é classificado, segundo Köppen, como Cfb. Os solos predominantes são Argissolo, Cambissolo Háplico e Neossolo Litólico. O uso predominante do solo é o cultivo de fumo, sendo que as lavouras ocupam 53 ha. O manejo do solo é um dos fatores determinantes para a variabilidade da produção de sedimentos na bacia. Os estudos de monitoramento hidrossedimentológico realizados na bacia, mostram uma produção relevante de sedimentos, com grande impacto sobre os recursos naturais (solo e água) (Merten \& Minella, 2005).

\section{Monitoramento hidrossedimentométrico}

O estudo da histerese foi realizado com dados obtidos na seção de monitoramento hidrossedimentométrico da bacia experimental, durante eventos significativos de precipitação. Para estudar o padrão de emissão de sedimentos na bacia foram escolhidos 19 eventos distintos que representassem diferentes magnitudes, diferentes épocas do ano e diferentes condições de uso e manejo do solo.

A determinação da vazão foi realizada a partir de leituras de nível efetuadas a cada 10 min por um linígrafo instalado numa calha Parshall, no exutório da bacia. A concentração de sedimentos em suspensão foi determinada coletando-se amostras do fluxo no riacho, com amostrador USDH48, durante os eventos e pelo monitoramento automático de turbidez. As medidas de turbidez são expressas em unidades nefelométricas de turbidez (NTU), utilizando-se uma curva-chave que correlaciona a CSS com a turbidez, estabelecida para o local de estudo (Minella et al., 2008). Para as amostras coletadas manualmente, a CSS foi determinada a partir do método da evaporação.
Conhecendo-se, então, a Q e a CSS, foram selecionados eventos que representassem diferentes condições para a construção do laço de histerese e para o cálculo do IH; para a interpretação dos resultados foram utilizados, também, os dados de precipitação de cada evento, uso e manejo do solo.

Em relação ao uso e manejo do solo foram selecionados eventos ocorridos nos anos de 2002, 2003 e 2004, anos em que o sistema de preparo do solo adotado pelos agricultores era o tradicional, que consiste no revolvimento total do solo sem medidas de conservação do solo. Em 2003 ocorreram, em resposta ao aumento do preço do fumo, mudanças significativas em relação ao uso do solo na bacia, sobretudo o aumento da área de cultivo, a drenagem das áreas úmidas e a retirada da mata ciliar (Merten \& Minella, 2007). A partir de 2004 a implementação gradual de medidas de conservação do solo e água aumentou significativamente as áreas sob manejo conservacionista, principalmente o uso de cultivo mínimo, com impactos positivos sobre a produção de sedimentos. No primeiro período a totalidade das lavouras era sob cultivo convencional e, em 2008, aproximadamente $80 \%$ das áreas apresentavam medidas de conservação do solo. Neste sentido foram selecionados eventos ocorridos em 2008, que representassem uma condição estável depois do início do uso de práticas conservacionistas, permitindo verificar se houve interferência nos padrões da CSS e da Q.

\section{RESULTADOS E DISCUSSÃO}

A partir dos eventos selecionados os gráficos de histerese foram construídos e sua forma analisada qualitativamente; na Figura 4 são apresentados alguns dos gráficos de histerese, obtidos no estudo.

A primeira evidência é que o sentido horário da histerese ocorreu em dezessete dos dezenove eventos medidos; apenas o evento 3 apresentou formato de sentido anti-horário e o evento 14, formato do tipo oito. Na Tabela 1 são apresentados alguns parâmetros que caracterizam as condições hidrológicas e sedimentológicas dos eventos monitorados e os índices de histerese IH.

Os principais fatores controladores do sentido do laço de histerese neste estudo foram as características fisiográficas da bacia, a pequena área de drenagem e as características das vertentes, que são declivosas e curtas, a proximidade das fontes de sedimentos com a rede de drenagem e o tipo de uso e manejo do solo. Apesar dos eventos apresentarem padrão semelhante no sentido do laço de histerese, ocorreu grande variabilidade nos valores calculados do IH; os eventos apresentaram valor médio de 3,21 com variação de $-0,12$ a 12,63, indicando que o processo predominante é o pico de sedimentos, antecedendo o pico de vazão mas com variabilidade dentro desta condição.

Para avaliar se os fatores controladores do IH estavam relacionados com o manejo do solo, os eventos foram separados em grupos com manejo distinto: antes de 2004, quando o manejo do solo foi convencional, com revolvimento do solo e após 2004, com manejo conservacionista com o uso de plantas de cobertura associadas ao cultivo mínimo. A produção de sedimentos monitorada entre os anos de 2002 e 2004 foi de $150 \mathrm{t} \mathrm{km}^{-2} \mathrm{ano}^{-1}$, a 


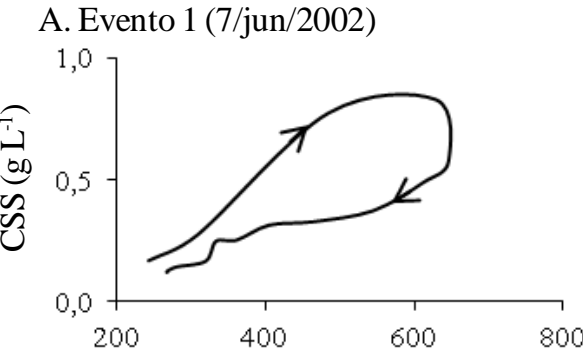

D. Evento 5 (30/abr/2003)

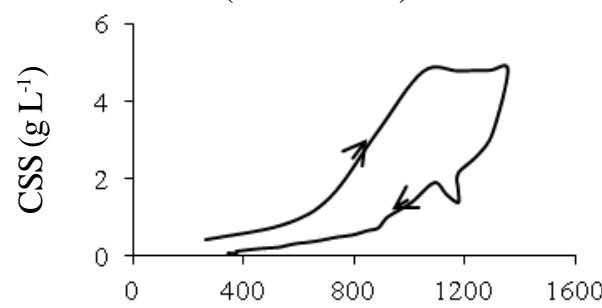

G. Evento $12(17 / \mathrm{ago} / 2008)$

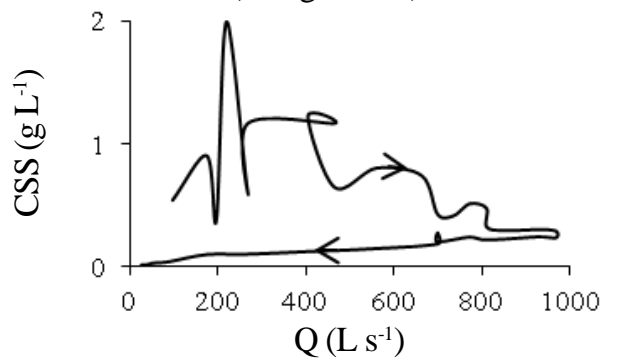

B. Evento 2 (21/ago/2002)

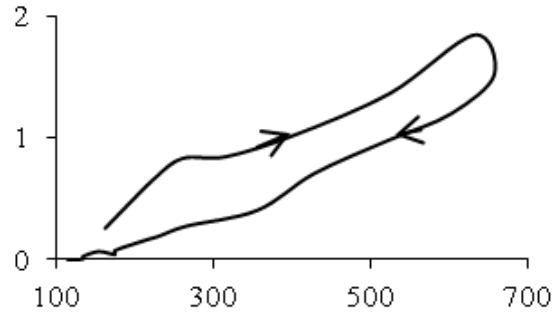

E. Evento 7 (2/mai/2008)

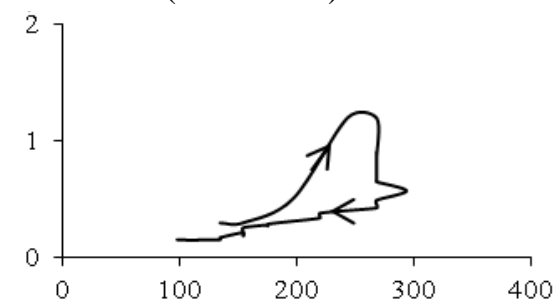

H. Evento 16 (15/out/2008)

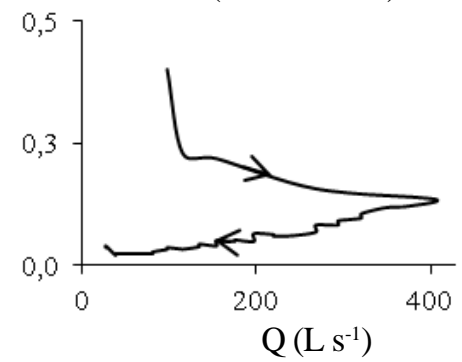

C. Evento 3 (8/out/2002)

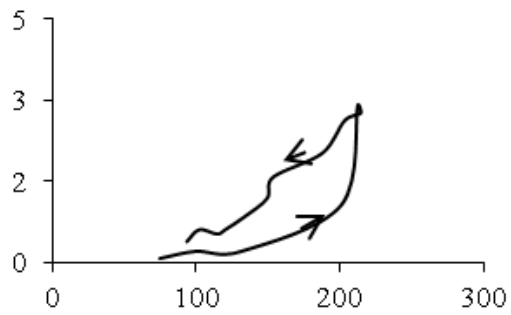

F. Evento 9 (26/jun/2008)

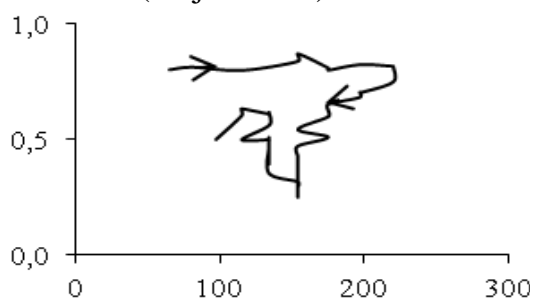

I. Evento 19 (2/dez/2008)

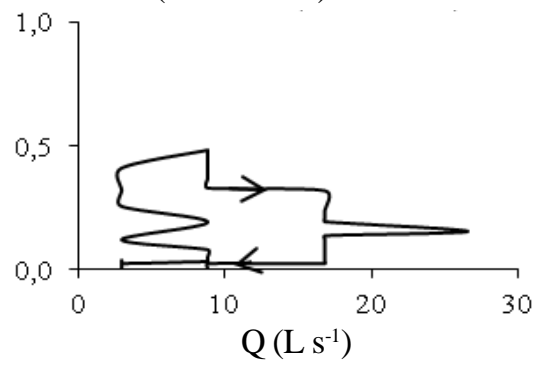

Figura 4. Laços de histerese para eventos na bacia

Tabela 1. Resultado das variáveis hidrossedimentológicas e da histerese, para os eventos monitorados na bacia de Arvorezinha*

\begin{tabular}{|c|c|c|c|c|c|c|c|c|c|c|c|}
\hline Evento & Data & $\mathbf{T}$ & P1 & P2 & $\mathbf{Q}_{\max }$ & $\mathbf{Q}_{\min }$ & $\mathbf{Q}_{\text {cen }}$ & CSS RC & CSS RD & IH & Histerese \\
\hline 1 & 7/jun/02 & 2,67 & 69 & 0 & 645 & 243 & 444 & 0,660 & 0,324 & 1,04 & $\mathrm{H}$ \\
\hline 2 & 21/ago/02 & 7,25 & 51 & 0 & 657 & 162 & 410 & 1,046 & 0,634 & 0,65 & $\mathrm{H}$ \\
\hline 3 & 8/out/02 & 2,17 & 16 & 3 & 215 & 75 & 145 & 0,353 & 1,071 & $-2,03$ & $\mathrm{AH}$ \\
\hline 4 & 20/jan/03 & 3,67 & 26 & 0 & 152 & 20 & 86 & 1,092 & 0,705 & 0,55 & $\mathrm{H}$ \\
\hline 5 & 30/abr/03 & 4,37 & 55 & 43 & 1355 & 265 & 810 & 2,475 & 0,578 & 3,28 & $\mathrm{H}$ \\
\hline 6 & 5/fev/04 & 2,43 & 49 & 17 & 495 & 25 & 260 & 4,651 & 0,363 & 11,82 & $\mathrm{H}$ \\
\hline 7 & 2/mai/08 & 110,33 & 53 & 28 & 293 & 134 & 213 & 0,758 & 0,329 & 1,31 & $\mathrm{H}$ \\
\hline 8 & 28/mai/08 & 77,67 & 60 & 0 & 175 & 9 & 92 & 1,728 & 0,302 & 4,72 & $\mathrm{H}$ \\
\hline 9 & 26/jun/08 & 138,83 & 30 & 29 & 219 & 65 & 142 & 0,831 & 0,340 & 1,45 & $\mathrm{H}$ \\
\hline 10 & 3/jul/08 & 71,83 & 34 & 0 & 219 & 17 & 118 & 0,843 & 0,301 & 1,80 & $\mathrm{H}$ \\
\hline 11 & 21/jul/08 & 21,83 & 29 & 3 & 51 & 3 & 27 & 1,101 & 0,109 & 9,07 & $\mathrm{H}$ \\
\hline 12 & 17/ago/08 & 83,83 & 84 & 0 & 966 & 9 & 487 & 0,691 & 0,144 & 3,80 & $\mathrm{H}$ \\
\hline 13 & 5/set/08 & 60,00 & 53 & 10 & 196 & 9 & 103 & 0,255 & 0,096 & 1,65 & $\mathrm{H}$ \\
\hline 14 & $29 /$ set/08 & 147,83 & 27 & 0 & 51 & 9 & 30 & 2,065 & 2,320 & $-0,12$ & 8 \\
\hline 15 & 11/out/08 & 96,67 & 16 & 0 & 404 & 9 & 206 & 0,792 & 0,109 & 6,29 & $\mathrm{H}$ \\
\hline 17 & 29/out/08 & 120,67 & 50 & 3 & 347 & 27 & 187 & 0,235 & 0,103 & 1,28 & $\mathrm{H}$ \\
\hline 18 & 8/nov/08 & 10,50 & 17 & 0 & 434 & 17 & 225 & 0,763 & 0,641 & 0,19 & $\mathrm{H}$ \\
\hline 19 & 2/dez/08 & 35,33 & 21 & 0 & 27 & 3 & 15 & 0,323 & 0,024 & 12,63 & $\mathrm{H}$ \\
\hline
\end{tabular}

* T - o tempo total do evento (h); P1 - a precipitação no dia do evento (mm); P2 - a precipitação no dia anterior ao evento (mm); $Q_{\max }$ - a vazão máxima do evento (L s-1); $Q_{\min }$ - a vazão inicial mínima do evento $\left(L s^{-1}\right) ; Q_{\text {cen }}$ - a vazão média calculada para o evento $\left(L s^{-1}\right) ; C S S R C$ - a concentração de sedimentos em suspensão para a $Q_{\text {cen }}$ no ramo crescente $\left(g L^{-1}\right) ; C S S$ RD - a concentração de sedimentos em suspensão para a $\mathrm{Q}_{\text {cen }}$ no ramo decrescente $\left(\mathrm{g} \mathrm{L}^{-1}\right)$; $\mathrm{IH}$ - a índice de histerese calculado para 0 evento; Histerese: $\mathrm{H}$ - 0 sentido horário, $\mathrm{AH}$ - 0 sentido anti-horário e 8 - 0 formato de oito

produção de sedimentos no ano de 2008 foi de $60 \mathrm{t} \mathrm{km}^{-2} \mathrm{ano}^{-1}$. Os resultados observados mostram que o valor médio de $\mathrm{IH}$ para $\mathrm{o}$ manejo conservacionista é maior $(\mathrm{IH}=3,5)$ do que o valor médio do IH para omanejo convencional ( $\mathrm{IH}=2,5)$; entretanto, a aplicação de uma análise estatística do tipo teste T de comparação entre médias mostra que a probabilidade de que as amostras dos dois grupos sejam da mesma população, é de 64,17\%.
A partir desses resultados verifica-se tendência de que, no período de manejo conservacionista ocorreu uma redução na CSS no ramo descendente do sedimentograma gerando valores de IH maiores; a partir de então, pôde-se inferir que no período em que foram incluídas as práticas de conservação de solo, houve uma redução da mobilização e aporte de sedimentos para a calha fluvial. 
Para avaliar o efeito da precipitação, os eventos foram separados segundo o volume de chuva precipitado no dia do evento, ou seja, um grupo com volumes superiores a $40 \mathrm{~mm}$ e outro com volumes inferiores a $40 \mathrm{~mm}$. Teoricamente, prevê-se que o valor de IH para os eventos de maior volume apresente um IH menor já que em eventos de maior pluviosidade existe maior suprimento de sedimentos. Os resultados mostram que para o grupo com volume maior que $40 \mathrm{~mm}$ o valor médio do índice de histerese foi de 3,12 e desvio padrão de 3,34 enquanto para o grupo de precipitação menor que $40 \mathrm{~mm}$ a média foi de 3,31 e o desvio padrão de 0,68 caso em que a aplicação da análise estatística do tipo teste $\mathrm{T}$ de comparação entre médias, mostra que a probabilidade de que as amostras dos dois grupos sejam da mesma população, é alta $(91,90 \%)$, ou seja, não há evidência estatística de que as amostras dos dois grupos sejam diferentes estatisticamente.

Em relação aos gráficos da Figura 4 e a Tabela 1 é possível fazer algumas inferências sobre as diferenças na histerese entre os eventos, a partir das características climáticas e de uso e manejo do solo:

i) Os eventos 1 e 9 ocorreram na época em que os solos são mobilizados para o plantio porém se diferenciam pelo tipo de preparo de solo utilizado. No evento 1 o preparo de solo utilizado foi o plantio convencional enquanto no evento 9 foi empregado o preparo conservacionista, que significa uma mobilização mínima do solo; a precipitação total dos eventos 9 e 1 foi semelhante porém a intensidade do evento 9 foi menor; para este caso observou-se que o IH foi maior no evento 9, efeito este atribuído à maior cobertura do solo condicionada pelo preparo conservacionista e, em parte, pela precipitação de menor intensidade.

ii) Os eventos 2 e 13 também ocorreram na época do preparo do solo para o plantio e com chuvas de alta intensidade; no evento 2 (preparo convencional) ocorreu maior escoamento superficial, além de produção de sedimentos, do que no evento 13 (preparo conservacionista); enfim, o IH foi menor no evento 2 mostrando o maior suprimento de sedimentos e capacidade de transporte.

iii) O evento 3 (preparo convencional), em comparação com os eventos 14, 15 e 18 (preparo conservacionista), também ocorreu na época de preparo do solo; entretanto, em período de chuvas de baixa intensidade; a primeira evidência é de que o evento 3 apresentou formato anti-horário e o evento 14, formato do tipo oito, os únicos que apresentaram comportamento diferente dos demais.

iv) Os eventos 4 e 19 ocorreram no início do verão quando a precipitação foi baixa; neste caso, os valores de IH foram muito diferentes entre os eventos; o evento 19, que ocorreu no período em que as práticas conservacionistas estavam sendo empregadas em grande parte da bacia e, no caso, o IH foi de 12,63 , enquanto para o evento 4 , que ocorreu durante o período em que as práticas de conservação de solos não haviam sido implantadas, o IH foi de 0,55 , diferença atribuída à maior disponibilidade de sedimentos deste primeiro período.

v) Nos eventos 5 e 12 as precipitações foram de alta magnitude embora o evento 12 tenha apresentado volume um pouco menor, porém com maior intensidade. Associado a esta condição, o evento 12 ocorreu em um período em que a cobertura do solo era menor; mesmo com características favoráveis à erosão do solo, o evento 12 apresentou um IH maior, demonstrando o efeito positivo de restringir o suprimento de sedimentos devido ao tipo de manejo do solo, que possibilitou uma cobertura maior de solo.

vi) Os eventos 6 e 16 apresentaram volumes de chuva semelhantes mas ocorreram em situações em diferentes épocas do ano. O evento 6 , constatado no período de plantio convencional, ocorreu em fevereiro, momento com alta cobertura do solo pelos resíduos do fumo e vegetação espontânea. Além disso, neste mês o solo se encontra com baixa umidade decorrente da alta evapotranspiração; assim, o IH apresentou um alto valor $(11,82)$ caracterizando uma condição de suprimento reduzido de sedimento no ramo descendente da curva; contrariamente, o evento 16 apresentou um IH baixo $(1,16)$, durante a primavera, que é o período em que as chuvas se concentram e os solos se encontram com umidade elevada, o que favorece não apenas a desagregação do solo mas também a transferência dos sedimentos para a calha fluvial.

vii) $\mathrm{O}$ evento 8 apresentou um volume total precipitado, semelhante aos eventos 1 e 2 ; entretanto, o valor de IH do evento $8(\mathrm{IH}=4,72)$ foi maior do que o dos eventos 1 e $2(\mathrm{IH}=$ 1,04 e $\mathrm{IH}=0,65$ respectivamente). Neste caso e pelo fato do evento 8 ter ocorrido com duração maior e, consequentemente, nesta condição o valor da vazão de pico $Q_{\max }$ foi menor, determinando menor capacidade de transporte de sedimentos.

viii) Os eventos 10 e 11 apresentaram precipitação semelhante porém o evento 10 apresentou maior valor de $\mathrm{Q}_{\text {max }}$, caso em que o IH encontrado foi sensivelmente menor ( $\mathrm{IH}=$ 1,8 para o evento 10 e $\mathrm{IH}=9,1$ para o evento 11 ), o que pode ser um indicativo da maior capacidade de transporte de sedimentos em eventos que geram o mesmo volume escoado mas com diferentes valores de $\mathrm{Q}_{\max }$.

Segundo Williams (1989), a ausência de um grande suprimento de sedimentos é, normalmente, a principal causa da ocorrência de efeito histerese de sentido horário em pequenas bacias. No caso deste estudo o pico de sedimentos adiantado em relação ao de vazão esteja relacionado, provavelmente, às características geomorfológicas da bacia e à configuração espacial de algumas componentes da paisagem, dentre os quais se destacam:

a. Terço superior da bacia, com menor declividade, e terço inferior com grande declividade, dificultando a transferência de sedimentos da região à montante e atrasando a onda da descarga líquida;

b. Bacia com alta declividade e em formato circular, que favorece a transferência de sedimentos;

c. Escoamento subsuperficial com grande importância nos terços superior e médio das encostas sob cultivo com exfiltração no terço inferior (pesquisa em andamento);

d. Grandes depósitos de sedimentos na base das encostas, rapidamente mobilizados para dentro do canal em eventos de maior magnitude;

e. Vales em forma de V, com pequenas planícies aluviais;

f. Conexão de estradas não pavimentadas, que cortam a rede de drenagem e contribuem, de forma significativa, para o aporte de sedimentos; 
g. Sistema de drenagem utilizado pelos agricultores para retirar o excesso de água na região, entre os camalhões de fumo;

h. Presença de sedimentos depositados no canal fluvial que são transferidos no início da onda de cheia nos eventos subsequentes;

i. Áreas ciliares degradadas e cultivadas que favorecem a transferência dos sedimentos das encostas para a calha fluvial.

Considera-se que o padrão do laço de histerese é governado pelos fatores acima mencionados, sendo que alguns deles (c e d) ainda são objeto de análise. É provável que o controle da magnitude dos valores de IH horário possa ser interpretado como uma sobreposição dos efeitos da variabilidade dos padrões da intensidade da precipitação (picos adiantados ou atrasados) e do uso e manejo do solo, especialmente da disponibilidade de sedimentos causados pelo movimento do solo e do índice de cobertura.

Sendo assim, pode-se inferir que o comportamento da forma do sedimentograma e do hidrograma de determinado evento, não pode ser definido simplesmente como função da área da bacia. Este complexo mecanismo de transferência depende, além das características da precipitação, do centro de massa das áreas que geram o escoamento e sedimentos (Klein, 1984), e da sua natureza (uso, manejo, relevo). É importante salientar que a Q e a CSS também sofrem influência direta dos processos de ligação entre calha e vertente e da variabilidade temporal das fontes de sedimentos que, por sua vez, está intimamente relacionada com as características da precipitação (Collins et al., 2010; Minella et al., 2007; 2008; Walling, 2005). O comportamento dos sedimentos suspensos nos rios é função, também, das condições de energia do escoamento superficial e da disponibilidade de energia para o seu transporte. Essas condições são extremamente variáveis entre os eventos, o que condiciona a processos de geração e transporte de sedimentos, também variáveis no tempo e no espaço.

A compreensão e a quantificação da histerese podem ser uma importante ferramenta para a utilização de modelos matemáticos de produção de sedimentos, sobretudo aqueles de escala de eventos como, por exemplo, Limburg Soil Erosion Model, haja vista que uma das principais dificuldades reside na discretização temporal do sedimentograma em conjunto com o hidrograma. Muitas vezes os modelos não são capazes de representar a defasagem no tempo entre o hidrograma e o sedimentograma, gerando uma superestimativa na produção de sedimentos, pois a concentração máxima de sedimentos coincide com a vazão máxima. Desta forma, a histerese é um fenômeno importante que deve ser contemplado nos estudos de modelagem como sendo um fenômeno que gera respostas sensivelmente diferentes na estimativa da produção de sedimentos, quando os modelos não são capazes de representar essa defasagem. A análise da histerese para diversos eventos pode fornecer um entendimento maior dos fatores controladores da relação CSS-Q.

\section{Conclusões}

1. O padrão de forma de histerese entre a Q e CSS para a bacia em estudo é do tipo laço horário, independente do uso do solo, manejo do solo e precipitação.
2. O índice quantitativo da histerese (IH) nos eventos dos dois períodos analisados apresentou um valor médio maior no período após a implementação de medidas de conservação do solo; entretanto, os valores de IH apresentam uma dispersão significativa.

3. Um número maior de eventos é necessário para a quantificação e separação da contribuição do controle da precipitação e do manejo do solo que afetam a variabilidade deste índice.

\section{Agradecimentos}

Ao Conselho Nacional de Desenvolvimento Científico e Tecnológico (CNPq) e ao Sinditabaco pelo apoio.

\section{LiTERATURA CITADA}

Bowes, M.; House, W.; Hodgkinson, R.; Leach, D. Phosphorus discharge hysteresis during storm events along a river catchment: the River Swale, UK. Water Research, Bygningstorvet, v.39, p.751-762, 2005.

Collins, A. L.; Walling, D. E.; Stroud, R. W.; Robson, M.; Peet, L. M. Assessing damaged road verges as a suspended sediment source in the Hampshire Avon catchment, southern United Kingdom. Hydrological Processes, v.24, p.1106-1122, 2010.

Goodwin, T. H.; Young, A. R.; Holmes, G. R.; Old, G. H.; Hewit, N.; Leeks, G. J. L.; Packman, J. C.; Smith, B. P. G. The temporal and spatial variability of sediment transport and yields with the Brasford Beck catchment, West Yorshire. The Science of the Total Environment, v.314-316, p.475494, 2003.

Horowitz, A. J. A quarter century of declining suspended sediment fluxes in the Mississippi River and the effect of the 1993 flood. Hydrological Process, v.24, p.13-34, 2009.

Jansson, M. B. Determining sediment sources areas in a tropical river basin, Costa Rica. Catena, v.47, p.63-84, 2002.

Klein, M. Anti clockwise hysteresis in suspended sediment concentration during individual storms. Catena, v.11, p.251257, 1984.

Lacombe, G.; Cappelaere, B.; Leduc, C. Hydrological impact of water and soil conservation works in the Merguellil catchment of central Tunisia. Journal of Hydrology, v.359, p.210-224, 2008.

Lawler, D. M., Petts, G. E.; Foster, I. D. L.; Harper, S. Turbidity dynamics and hysteresis patterns during spring storm events in an urban headwater system: The Upper Tame, West Midlands, UK, Science of the Total Environment, v.360, p.109-126, 2006.

Lefrancois, J.; Grimaldi, C.; Gascuel-Odoux, C.; Gilliet, N. Suspended sediment and discharge relationships to identify bank degradation as a main sediment source on small agricultural catchments. Hydrological Processes, v.21, p.2923-2933, 2007. 
Lenzi, M. A.; Lorenzo, M. Suspended sediment load during floods in a small stream of the Dolomites (northeastern Italy). Catena, v.39, p.267-282, 2000.

Merten, G. H.; Minella, J. P. G. Impact on sediment yield due to intensification of tobacco production in a catchment in southern Brazil. In: Walling, D. E.; Horowitz, A. J. (ed.). Sediment budgets, v.292, n.2. Wallinford: IAHS Press, 2005. p.239-244.

Minella, J. P. G.; Merten, G. H.; Clarke, R. T.; Reichert, J. M. Estimating suspended sediment concentrations from turbidity measurements and the calibration problem. Hydrological Processes, v.22, p.1819-1930, 2008.

Minella, J. P. G.; Merten, G. H.; Reichert, J. M.; Santos, D. R. Identificação e implicações para a conservação do solo das fontes de sedimentos em bacias hidrográficas. Revista Brasileira de Ciência do Solo, v.31, p.1637-1646, 2007.

Minella, J. P. G.; Merten, G. H.; Walling, D. E.; Reichert, J. M. Changing sediment yield as an indicator of improved soil management practices in southern Brazil. Catena, v.79, p.228236, 2009.

Minella, J. P. G.; Walling, D.; Merten, G. Combining sediment source tracing techniques with traditional monitoring to assess the impact of improved land management on catchment sediment yields. Journal of Hydrology, v.348, p.546-563, 2008.

Morgan, R. P. C. Soil erosion and conservation. Oxford: Blackwell Publishing, 2005. 198p.
Nyssen, J.; Clymans, W.; Poesen, J.; Vandecasteele, I.; Baets, S.; Haregeweyn, N.; Naudts, J.; Hadera, A.; Moeyrsons, J.; Haile, M.; Deckers, J. How soil conservation affects the catchment sediment budget - A comprehensive study in the north Ethiopian highlands. Earth Surface Process and Landforms, v.34, p.216-1223, 2009.

Orwin, J. F.; Smart, C. C. The evidence for paraglacial sedimentation and its temporal scale in the deglacierizing basin of Small River Glacier, Canada. Geomorphology, v.58, p.175-202, 2004.

Seeger, M.; Errea, M.; Begueria, S.; Arnaez, J.; Marti, C.; GarciaRuiz, J. Catchment soil moisture and rainfall characteristics as determinant factors for discharge/suspended sediment hysteretic loops in a small headwater catchment in the Spanish pyrenees. Journal of Hydrology, v.288, p.299-311, 2004.

Stott, T. Impacts of constructing a rural cycle way on suspended sediment transport processes. Catena, v.68, p.16-24, 2006.

Walling, D. E. Linking land use, erosion and sediment yield in river basins, Hydrobiology, v.410, p.223-240, 1999.

Walling, D. E. Tracing suspended sediment sources in catchments and river systems. Science of the Total Environment, v. 344, p.159-184, 2005.

Walling, D. E. The changing sediment loads of the world's rivers. Annals of Warsaw of Life Sciences - SGGW. Land Reclamation, v.39, p.3-20, 2008.

Williams, G. P. Sediment concentration versus water discharge during single hydrologic events in rivers. Journal of Hydrology, v.111, p.89-116, 1989. 